\title{
Financing Strategies For A Nuclear Fuel Cycle Facility
}

\author{
David Shropshire \\ Sharon Chandler
}

December 2005

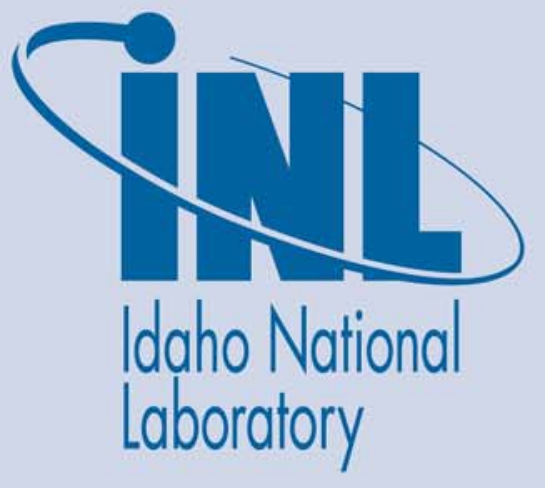

The INL is a U.S. Department of Energy National Laboratory operated by Battelle Energy Alliance 

INL/EXT-05-01021

\title{
Financing Strategies For A Nuclear Fuel Cycle Facility
}

\author{
David Shropshire
}

Sharon Chandler - Georgia Institute of Technology, Graduate Fellowship to INL

December 2005

\section{Idaho National Laboratory \\ Idaho Falls, Idaho 83415}

Prepared for the

U.S. Department of Energy

Office of Nuclear Energy

Under DOE Idaho Operations Office

Contract DE-AC07-05ID14517 

December 16, 2005

\section{ABSTRACT}

To help meet the nation's energy needs, recycling of partially used nuclear fuel is being considered more and more as a necessary step in a future nuclear fuel cycle, but incorporating this step into the fuel cycle will require considerable investment. This report supports an ongoing evaluation of financing scenarios for recycling facilities integrated into the nuclear fuel cycle. A range of options from fully government owned to fully private owned were evaluated in FY-05 using a deterministic analysis. In FY-06 this analysis is being extended by development of an economic tool that captures the financing algorithms and supports the decision analysis capabilities needed to effectively manage and exploit uncertainty and complexity. The analysis performed in this report utilized DPL (dynamic programming language 6.0), which can systematically optimize outcomes based on user-defined criteria (e.g., lowest life-cycle cost, lowest unit cost).

This evaluation concludes that the lowest unit costs and lifetime costs follow a fully government-owned financing strategy, due to government forgiveness of debt as sunk costs. However, this does not mean that the facilities should necessarily be constructed and operated by the government. The costs for hybrid combinations of public and private (commercial) financed options can complete under some circumstances with the government option. This analysis shows that commercial operations have potential to be economical, but there is presently no incentive for private industry involvement, due to the current legal framework of the Nuclear Waste Policy Act, which establishes government ownership of partially used commercial nuclear fuel.

Overwhelmingly, uncertainty in annual facility capacity led to the greatest variations in unit costs necessary for recovery of operating and capital expenditures; the ability to determine annual capacity will be a driving factor in setting unit costs. For private ventures, the costs of capital, especially equity interest rates, dominate the balance sheet; and the annual operating costs, forgiveness of debt and overnight costs dominate the government case. The uncertainty in operations, leading to lower than optimal processing rates, is the most detrimental issue economically. Conversely, lowering debt interest rates and the required return on investments can reduce costs for private industry.

Additional studies on facility ownership will be researched and incorporated into the 2006 AFC Cost Basis report. The authors are aware of similar studies on MOX fuel fabrication facilities (ORNL 1996) for weapons

plutonium disposition. These studies showed that government ownership lead to the lowest life cycle and unit costs. For the U.S. MOX Fabrication Facility, now under construction at the Savannah River site, government funds are being used for construction; however, a private company will operate the plant with performance-based incentives. Also additional analysis will be performed to better understand facility capacity cost sensitivities and other high cost impact items. 
December 16, 2005 


\section{CONTENTS}

ABSTRACT

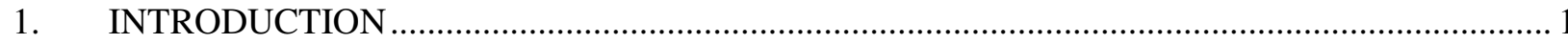

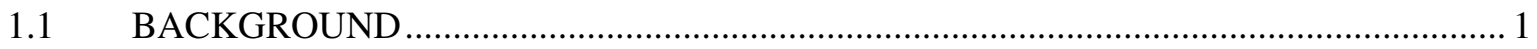

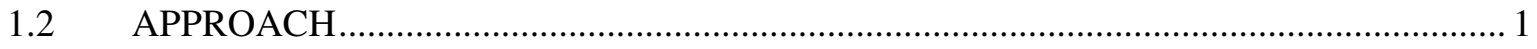

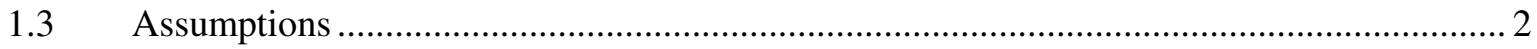

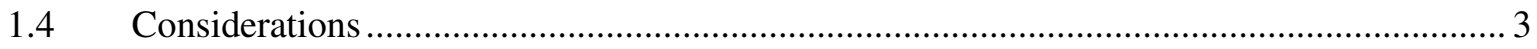

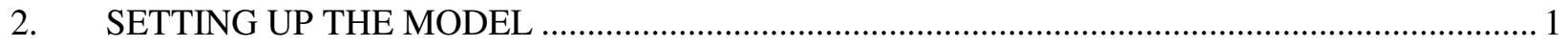

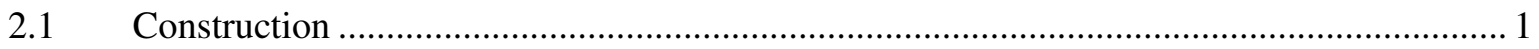

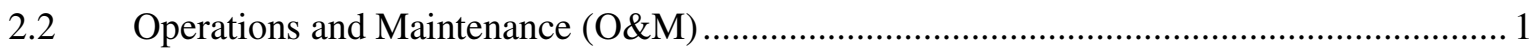

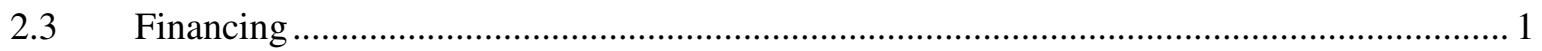

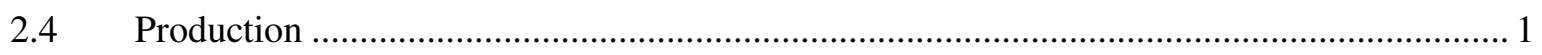

2.5 Evaluation of Major Uncertainties ....................................................................... 1

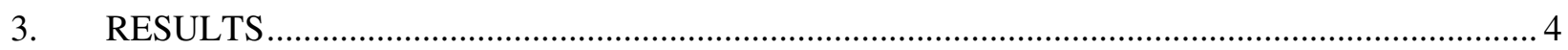

W.1 What if the government doesn't forgive the debt? ........................................................ 5

3.2 What if operational problems cause the capacity to become less than nominal? ................ 7

3.3 What if guaranteed contracts or government incentives lead to lower risk for private

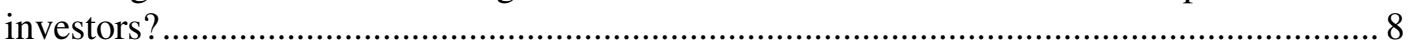

3.4 How does this analysis incorporating elements of variable uncertainty compare to the original AFCI deterministic analysis? ..................................................................... 10

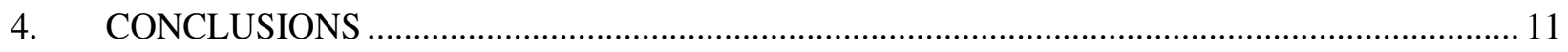

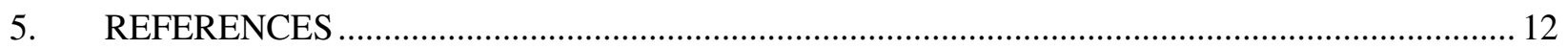

\section{FIGURES}

Figure 1. Model for financing a generic fuel cycle facility.......................................................... 1

Figure 2. Tornado diagram showing the effect of uncertainty on unit costs......................................... 2

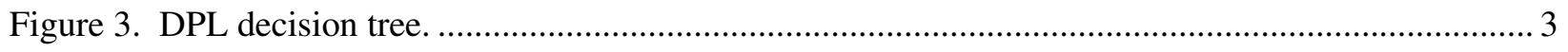




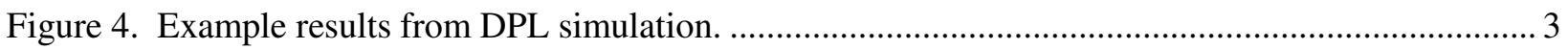

Figure 5. Estimated unit costs for each financing strategy. ............................................................. 4

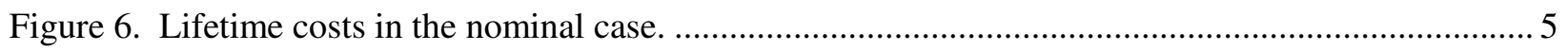

Figure 7. Estimated unit costs in the government-not-forgiving-debt simulation. ................................... 6

Figure 8. Estimated lifetime costs in government-not-forgiving-debt simulation................................... 6

Figure 9. Estimated unit costs in $30-70 \%$ annual capacity simulation............................................... 7

Figure 10. Estimated lifetime costs in $30-70 \%$ annual capacity simulation........................................ 8

Figure 11. Estimated unit costs in lower risk simulation..............................................................

Figure 12. Estimated lifetime costs in the lower risk simulation..................................................... 10

\section{TABLES}

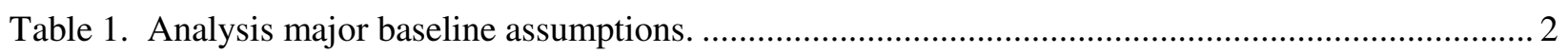

Table 2. Financial assumptions used in the model....................................................................... 2 


\section{Financing Strategies for a Nuclear Fuel Cycle Facility}

\section{INTRODUCTION}

With the current heightened concern for air pollutants and natural resource conservation, there is a focus on future energy options. One option is the resurgence of nuclear power with a new, closed fuel cycle. Research in this area is being conducted within the framework of the advanced fuel cycle initiative (AFCI). "A mission of the AFCI program is to develop technologies that concurrently will meet the need for an economic and sustained nuclear option while satisfying requirements for a controlled, proliferationresistant nuclear material management system" (AFCI 2005).

Initial work on the economics of the private sector versus regulated nuclear fuel cycle facilities was completed by Chaim Braun in 2005 and was reported in the 2005 Advanced Fuel Cycle Cost Basis, Appendix D (Shropshire 2005). The purpose of this paper is to extend the single point economic analysis performed by Chaim Braun to a probability and uncertainty analysis sphere. To support these advanced calculations a dynamic model was created using dynamic programming language (DPL) Version 6.0 Professional. Scenarios were run to determine which factors were most influential in determining benefits and costs, and then evaluated to develop a realistic range of values. With this range, both discrete value and Monte Carlo simulations were run and the scenarios evaluated. This report discusses their merits and attempts to understand the cost implications from facility ownership options: government, private, and combinations of government/private.

\subsection{BACKGROUND}

There is little directly applicable research on the ownership options for a first-of-a-kind recycling facility. Initial work performed by the National Academy of Sciences (NAS) evaluated recycling facility ownership options for a private company, a utility consortium, and the government (NAS 1996). The NAS determined that "A pure, private venture to design, build, own, and operate such a complex, without government financial guarantees, appears unrealistic." Further, they conclude, "At a minimum, it would appear that some new type of government risk/cost sharing, far more extensive than on past projects/programs, would be necessary to attract utility participation." A comparative analysis of a generic fuel cycle facility was conducted by Braun for the AFCI Economic Benefits activity, analyzing the unit costs for recycling under various ownership options with an ultimate conclusion that government ownership led to lowest costs, especially if the government were willing to forgive debt. Braun also explored qualitative differences in financing options, such as the national social benefit of such a facility and government legal obligations to the utilities toward partially used fuel disposition. In addition, there has been some comparative economic analysis done on public and private facilities in the past; however, most of these are not wholly representative in this case, as the bulk of these analyses are based on the electric generating industry power plants and carry assumptions not applicable here. Most useful for the AFCI's purposes is the historical evidence of private industry having lower operating costs compared to similar government facilities (Kwoka 2005).

\subsection{APPROACH}

This analysis is designed to meet the needs of the AFCI program. To that end, we have considered the required capacities and processes stated in the AFCI Report to Congress (AFCI 2005). The AFCI program is considering advancements to the established PUREX process, such as UREX+ and pyroprocessing as recycling technology options. The specific designs and methods for separation in a future fuel recycle facility have not yet been determined. There is limited cost data available on new recycle facility costs that would be applicable to a United States application. The AFCI program has 
compiled historical reports and studies on recycling, and has determined that there are very large cost uncertainty ranges for these facilities.

The current plan does not anticipate large numbers of recycle facilities being built, so the relative advantages coming from next-of-a-kind (Nth-of-a-kind or NOAK) facilities are ignored, and the financing options are only considered for the first facility. Subsequent facilities, if built, will likely have similar financing considerations as the first.

This model follows the conclusions drawn from independent review of the literature discussing recycle facilities, especially those cited in Section 1.1, Background. For comparison, this model could easily be modified to model other related facilities, such as those intended for fuel fabrication or combined (integrated) recycling and refabrication. The ownership structure for facilities in mature industries is not in doubt, but similar analyses may be necessary for other new processes that accompany or complement the desire to recycle partially used nuclear fuel.

The model was set up from the variables and data from the AFCI's previous work in FY-05. The financing options were (a) totally government-constructed-and-operated facility, (b) a governmentconstructed facility sold to a private sector corporation with a portion of initial investment forgiven, (c) a private-sector regulated facility, and (d) private-sector non-regulated facility. This type of economic model could be extended and easily modified to accommodate all facilities to be considered (e.g., separations, fabrication, storage, etc).

\subsection{Assumptions}

The major baseline financial assumptions underlying this analysis are provided in Tables 1 and 2 . These assumptions are consistent with the analysis of economics of the private sector versus regulated nuclear fuel cycle facility reported in the 2005 AFC Cost Basis report, Appendix D (Shropshire, 2005). All the computations carried out here relate to a hypothetical facility and do not represent any specific plant design or cost data.

Table 1. Analysis major baseline assumptions.

\begin{tabular}{ll}
\hline & Baseline values used in all four cases \\
\hline Total capital charges (TCIC) & \$8.0 Billion \\
Annual operating costs (O\&M) & \$396 Million/year \\
Annual capacity & 800,000 MTHM/year) \\
Lifetime & 30 Years \\
\hline
\end{tabular}

Table 2. Financial assumptions used in the model.

\begin{tabular}{lcccc}
\hline & $\begin{array}{c}\text { Government } \\
\text { Constructed } \\
\text { \& Operated }\end{array}$ & $\begin{array}{c}\text { Government } \\
\text { Constructed } \\
\text { \& Sell Private }\end{array}$ & $\begin{array}{c}\text { Regulated } \\
\text { Private }\end{array}$ & $\begin{array}{c}\text { Nonregulated } \\
\text { Private }\end{array}$ \\
\hline Debt portion (\%) & 100.0 & 40 & 50 & 30 \\
Debt interest rate (\%/yr) & 4.0 & 4.8 & 4.8 & 9.0 \\
Equity portion (\%) & 0 & 60 & 50 & 70 \\
Required return on investment (\%/yr) & NA & 10.5 & 8.5 & 16.0 \\
Insurance (\%/yr) & 2.0 & 2.0 & 2.0 & 2.0 \\
Federal taxes (\%/yr) & NA & 33.0 & 33.0 & 33.0 \\
State and local taxes (\%/yr) (average) & NA & 5.0 & 5.0 & 5.0 \\
\hline
\end{tabular}




\begin{tabular}{lllll}
\hline Forgiveness of Plant Investment (\%) & $100 \%$ & $62.5 \%{ }^{\mathrm{b}}$ & NA & NA \\
\hline
\end{tabular}

\subsection{Considerations}

Originally, return on investment (ROI) in the model was a calculated variable from other inputs, but this setup did not allow direct comparison of government and private facilities. From these financial assumptions, the ROI to private investors becomes fixed in the model at the required ROI (as shown in Table 2). This may not be the real-world scenario, as investors may demand a higher ROI than suggested here. Also, private companies may choose to allocate capital expenses differently among debt and equity portions. There are some tax advantages to maximizing debt; however, there has not been the tendency of corporations to simply maximize debt, because there are other advantages to having equity financing.

To meet the needs of a new generation of nuclear power plants, the first commercial-scale recycling facility would be employed by the year 2025, with a capacity of 2,500 to 3,000 MT/year and a design life of 40 years (AFCI 2005). Further, AFCI is also considering 3,000 MT/ year facilities with a lifetime of 60 years; these are not modeled here. These capacities suggest significant deviations from the currently available data on other existing recycle facilities and estimates of future scenarios. The existing technology (PUREX) is used at both La Hague (2 with $800 \mathrm{MT} / \mathrm{yr}$ each) and THORP (1,200 MT/yr). This analysis uses the $800 \mathrm{MT} / \mathrm{yr}$ as a baseline capacity for comparison. We investigate the implications from increasing this capacity to those under consideration by the AFCI Program.

From a systems perspective, to achieve the lowest nuclear energy costs we have to sharpen our pencils to find ways to reduce the cost for fuel recycling. In seeking the lowest cost options we have to consider the impacts from recycling from various perspectives. One perspective is that of the utility that would need to accept recycled fuel for use in their reactors. Another perspective is that of a waste manager that would need to disposition costs of the recycled streams (i.e., placement into acceptable waste forms, packaging, transportation, and disposal). The focus of this report is on optimizing the facility costs, but the AFCI program is also evaluating the recycling costs from the other perspectives. Additionally, recycling costs need to be compared against other fuel processing and technology costs, and with competing energy costs when determining energy strategies.

\footnotetext{
${ }^{\mathrm{b}}$ Of the debt, $62.5 \%$ is considered forgiven by the government. The private owner then distributes capital portions over only the $37.5 \%$ of the initial capital investment made by the government.
} 


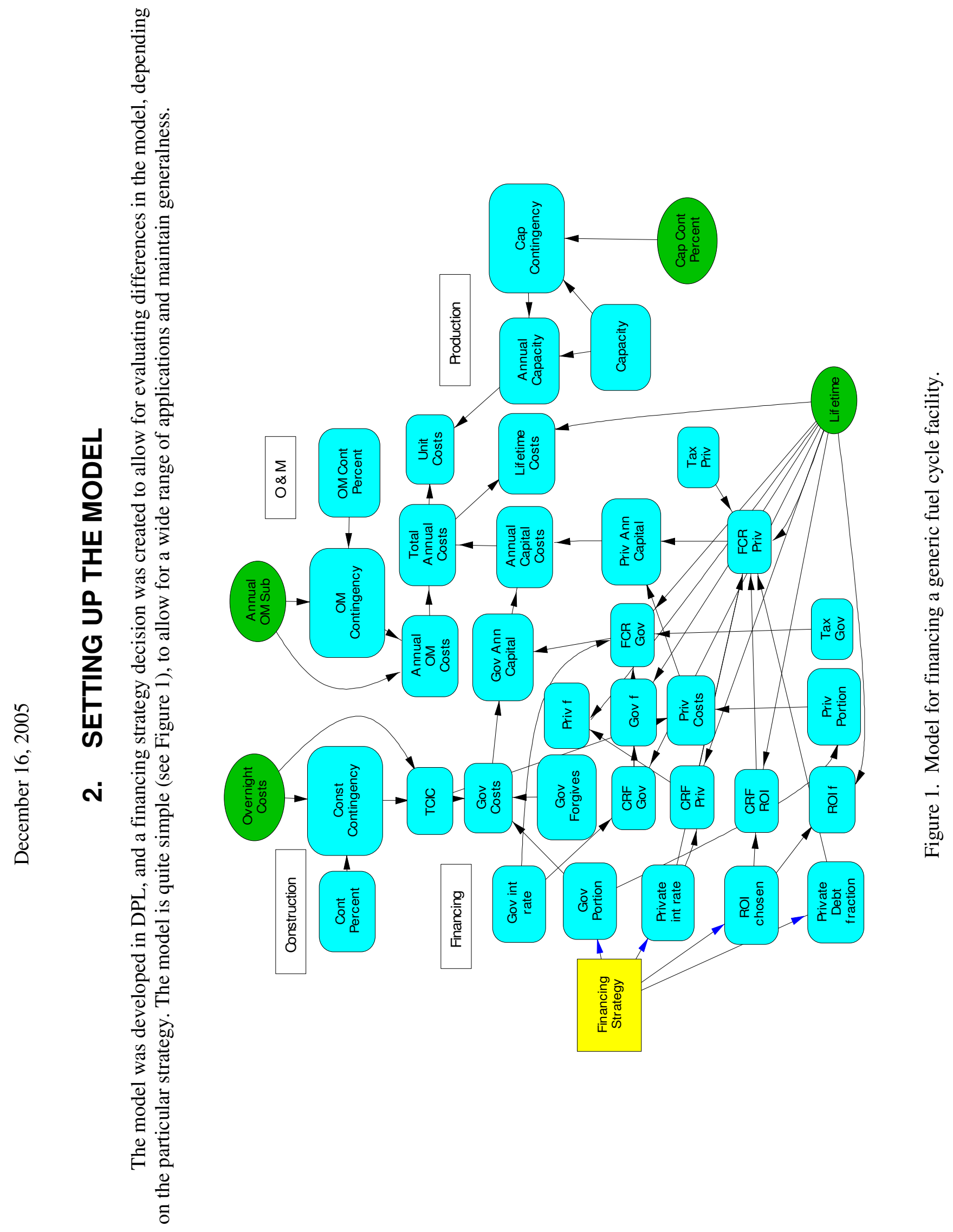




\subsection{Construction}

The construction costs have not been broken down into specifics, but the model has the flexibility to do so. The construction section of the model calculates the overnight costs plus a contingency. The contingency rate is based on historical experience and design maturity. The relationship between the contingency and annual capital costs is explained in Section 2.3, Financing. Initially the contingency costs are set at zero.

\subsection{Operations and Maintenance (O\&M)}

The O\&M costs are structured the same as the construction costs, with a central O\&M cost defined, and then a percentage contingency (based on historical experience and/or design maturity) is added to arrive at annual O\&M costs. The O\&M costs were defined at a fixed rate of $\$ 396 \mathrm{M} /$ year for all four scenarios.

\subsection{Financing}

The financing portion of the model is more complex, and supports the most important aspect of the model. From "financing strategy" (yellow decision box in Figure 1), the model adjusts: the portion of debt carried by the government, private interest rate, chosen ROI, and private debt fraction. These variables are adjusted to meet the various scenarios in the table shown above in Section 1.3, Assumptions. Using these values, the model calculates the fixed charge rate (FCR), Equation (1), for each option. Detailed explanations of calculations can be found in financing textbooks and the Cost Basis report (Shropshire, 2005).

$$
\begin{aligned}
\mathrm{FCR}= & \left((1-\mathrm{Tax})^{\wedge}-1\right)^{*}\left(\left(\text { Equity Fraction* } \mathrm{CRF}_{\text {equity }}\right)-\mathrm{Tax} /\right. \text { Lifetime } \\
& +\left(\text { Debt Fraction* }\left(\mathrm{CRF}_{\text {debt }}-\operatorname{Int} / \mathrm{Tax}\right)\right) .
\end{aligned}
$$

The annual capital charge is simply the TCIC multiplied by the FCR.

Annual Capital Costs $=$ TCIC $*$ FCR

Total annual costs include the annual capital costs plus the annual O\&M costs. These will necessarily vary by ownership option.

\subsection{Production}

The production section consists of the annual throughput and contingency on capacity. This contingency is calculated as a percentage decrement from given capacity. Initially the contingency is set to zero.

Unit costs are determined by ensuring that all annual costs are met with the proceeds from the throughput:

Unit Cost $=$ Total Annual Costs $\div$ Annual Capacity .

\subsection{Evaluation of Major Uncertainties}

Before we can begin modeling this problem, we must determine which variables will offer significant change to the end result, unit cost. In DPL, the sensitivity of each variable is determined by creating a tornado diagram. The variables for the baseline government constructed \& operated option were run over a range of potential values as noted on the value tornado diagram in Figure 2. Only those 
variables that had a significant effect on the value of the estimated unit costs were included in this analysis.

\section{Baseline Government Constructed \& Operated}

\section{Expected Value $=800,000 \mathrm{kgHM} / \mathrm{yr}, \$ 396 \mathrm{M} / \mathrm{yr}$, Forgive 0\% Debt, $\$ 8,000 \mathrm{M}, 30$ yrs life}

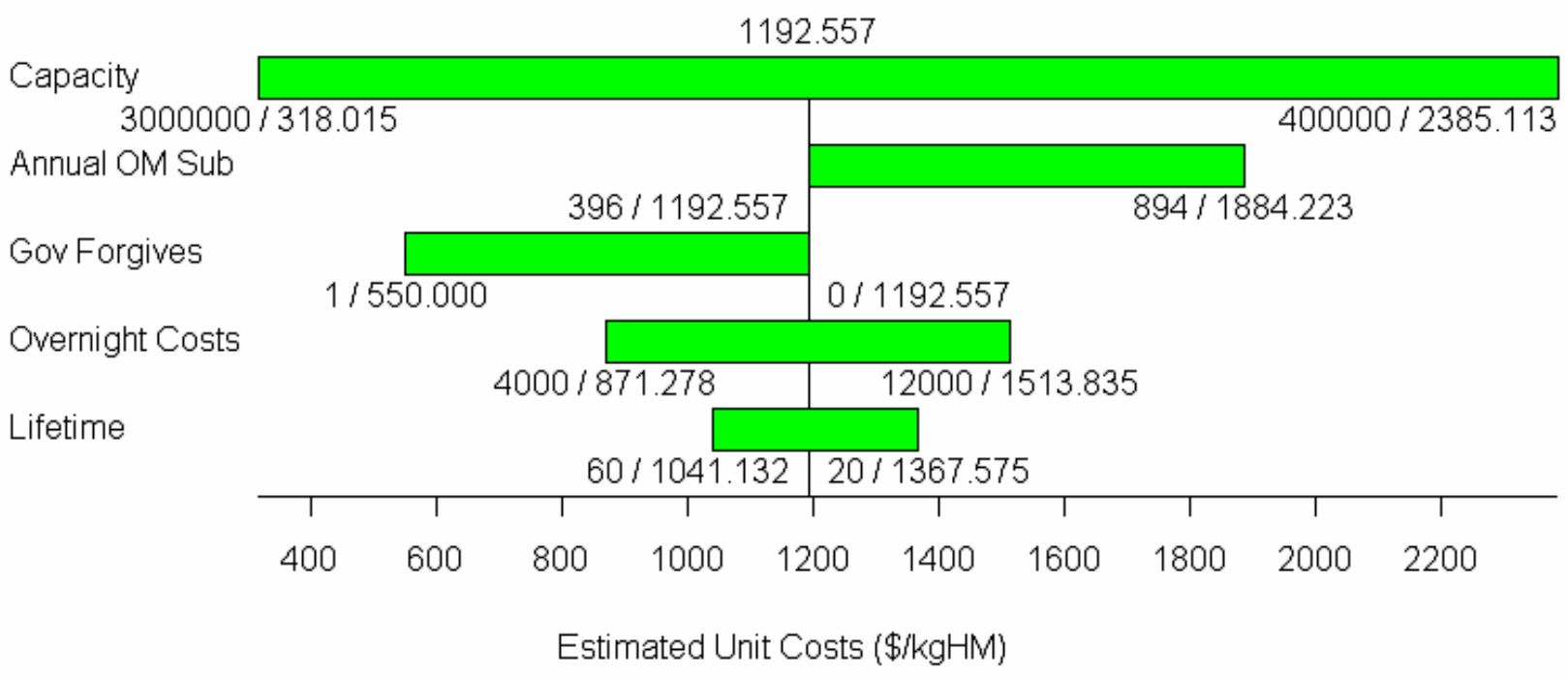

Figure 2. Tornado diagram showing the effect of uncertainty on unit costs.

Clearly, annual capacity is the most dominant variable on the calculated unit cost. If high capacities (approaching 3,000 MT/year) are obtained within the same capital and operation cost parameters as modeled, then unit costs as low as $\$ 500 / \mathrm{kgHM}$ could be achieved. These capacities could result from significant economies of scale (specifically capital cost versus capacity) arising from the construction design and facility operations designed for optimal economic throughput.

Reduction of overnight costs can also directly improve the costs for reprocessing. A reduction of the overnight costs by $50 \%$ has an impact of reducing the unit costs by a third. Annual operations and maintenance favors operation by the government or government/private sector over the higher costs expected from a regulated facility. Government forgiveness of the original investment can result in a unit cost reduction of up to $25 \%$, for a government facility that claims $100 \%$ sunk cost. The lifetime of the facility has about a 5\% impact on the unit costs, but in combination with higher facility capacities could help to defer the need for additional recycle plants.

Now that we understand the effect of changing values one-at-a-time, we can add uncertainty to the variables and run the model in the form of a multivariable sensitivity study. Uncertainty has been added to the model by assigning values in the forms of normal curves with a mean at the nominal value and a standard deviation of $10 \%$. This results in $99.73 \%$ of possible input values falling into the range of three standard deviations on either side of the nominal value. Those variables that have added uncertainty are shown in the diagram above. One exception to the normal curve is lifetime. The lifetime could vary substantially from the 30 year assumption, so the uncertainty in lifetime is modeled by a uniform probability from 20 to 60 years. Also, the amount of debt incurred by the government which is forgiven is assumed to be $100 \%$, except in the case where it is modeled that the government does not forgive (or sink) debt. 
These uncertainties are added to the model by changing the input variable into a continuous chance (stochastic) node. Continuous chance nodes are shown as green ovals in the model. Constant input variables and calculations are shown by blue rectangles in Figure 1.

The way the DPL program runs through the scenarios is depicted by the decision tree in Figure 3 . The decision tree shows the flow through the model to determine the outcomes. The model will run through each of the four financing options with 1 million cases; each case will go through the decision tree, so we actually run 4 million cases each time we do an analysis on this model. The case will stop at each continuous chance node, where the model will "roll the dice" (Monte Carlo simulation) to determine a value for that parameter; then the model will continue until the desired value for comparison is reached. In this case, the objective function is unit costs, which is evaluated and then compared across the financing scenarios.

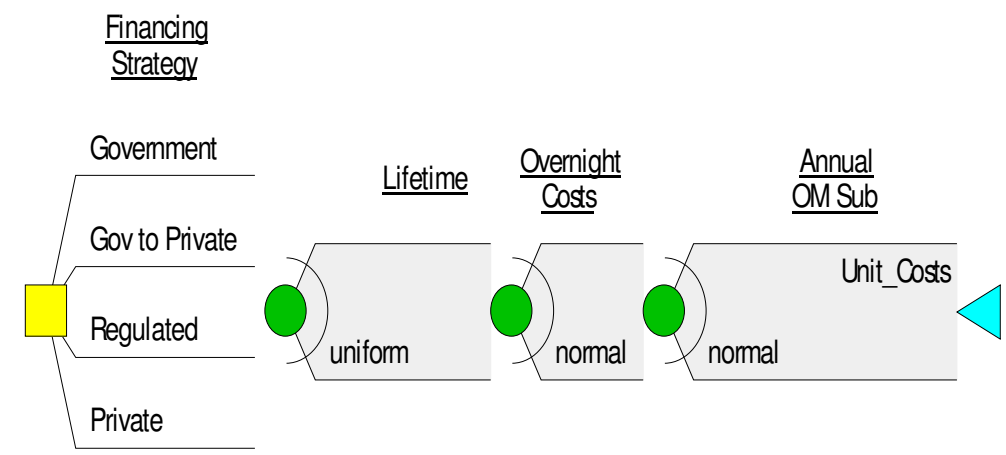

Figure 3. DPL decision tree.

As a result of the simulation, the expected values for each of the financing strategies are displayed. The financing strategy with the lowest unit cost is identified in the bolded top line of Figure 4 . In this example the government option returned the lowest unit cost at $\$ 550 / \mathrm{kgHM}$, based on the uncertainty values used in this run. As various assumptions are tested and the uncertainty distributions modified (as we have done in the subsequent analysis in this report) the expected values will change and the lowest cost financing strategy can shift between strategies. The results of the simulation lead us toward identifying the best financing option under complex circumstances.

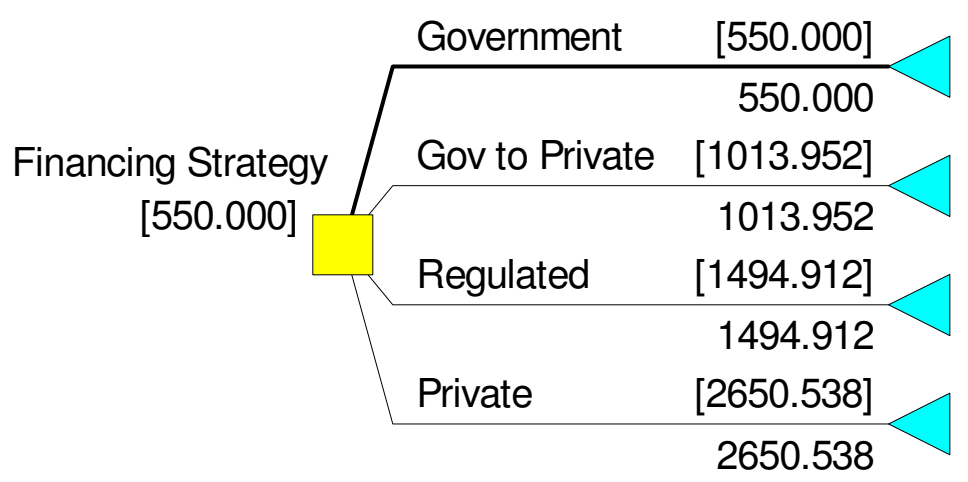

Figure 4. Example results from DPL simulation. 


\section{RESULTS}

With this setup, the model was run to see the range of unit costs and the relationship between the financing strategies. For clarification, these simulations are all run under the assumptions documented in the previous section, except where explicitly noted. From Figure 5, we can see that there is a large distribution of costs from the low of nearly $\$ 500 / \mathrm{kgHM}$ to around $\$ 2600 / \mathrm{kgHM}$.

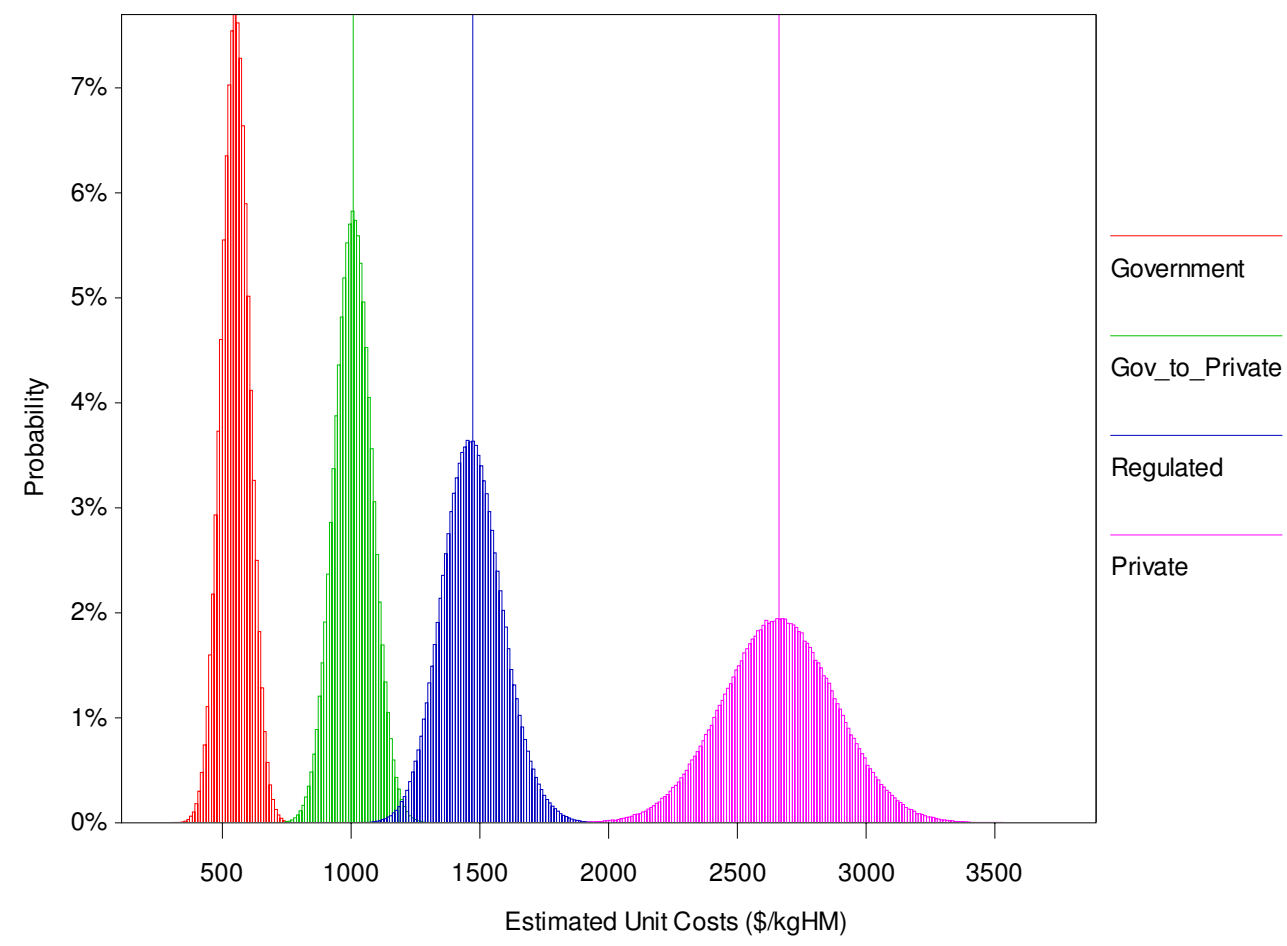

Figure 5. Estimated unit costs for each financing strategy.

The government option provides the lowest estimated unit cost distribution. By decreasing the government involvement, the options become progressively more expensive. The regulated option is more attractive than private ownership, even though none of the debt is forgiven. The private (non-regulated) option is clearly the most expensive option. 


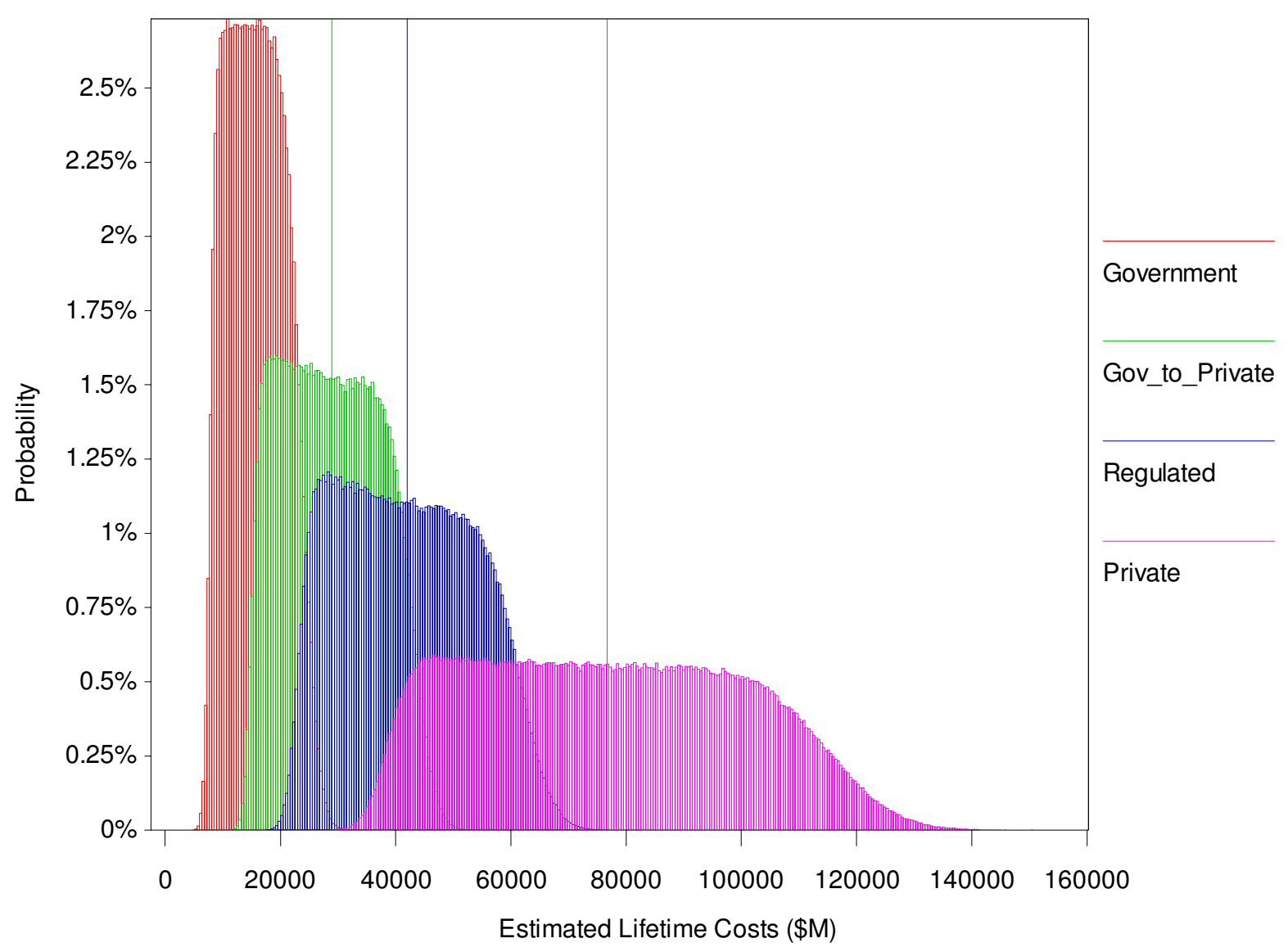

Figure 6. Lifetime costs in the nominal case.

Figure 6 shows a broad and overlapping range of estimated lifetime costs across the options (representing 20 to 60 years of operation). All costs are considered recovered, as the unit costs are set by the facility costs. The government option results in the lowest costs primarily due to the forgiven debt of $\$ 8$ billion, and the government-to-private option is the next least cost, as $62.5 \%$ of the initial investment is forgiven and not recovered from the sales of services.

\subsection{What if the government doesn't forgive the debt?}

The public is more likely to support a project that will minimize government-sunk money. Historically, it has been the practice of the U.S. Government to forgive its own debt when building facilities that are for the public good. Politically, the term public good varies in definition by users; so, what is a public good for some may be a waste to others. For these reasons we consider cases that minimize government-sunk money by repaying the debt over the facility's lifetime.

In this simulation, only the wholly government option is changed, to reflect the option that the government could choose to not forgive the debt. When debt is not forgiven, the government cost advantage is significantly diminished over the course of the lifetime of the facility, as shown in Figures 7 and 8. In this case, the government is assumed to still forgive $62.5 \%$ of the debt when transferred to the private sector, which results in reduced costs to the taxpayers. This change results in the government-tothe-private option becoming slightly less expensive than the wholly government option. 
December 16, 2005

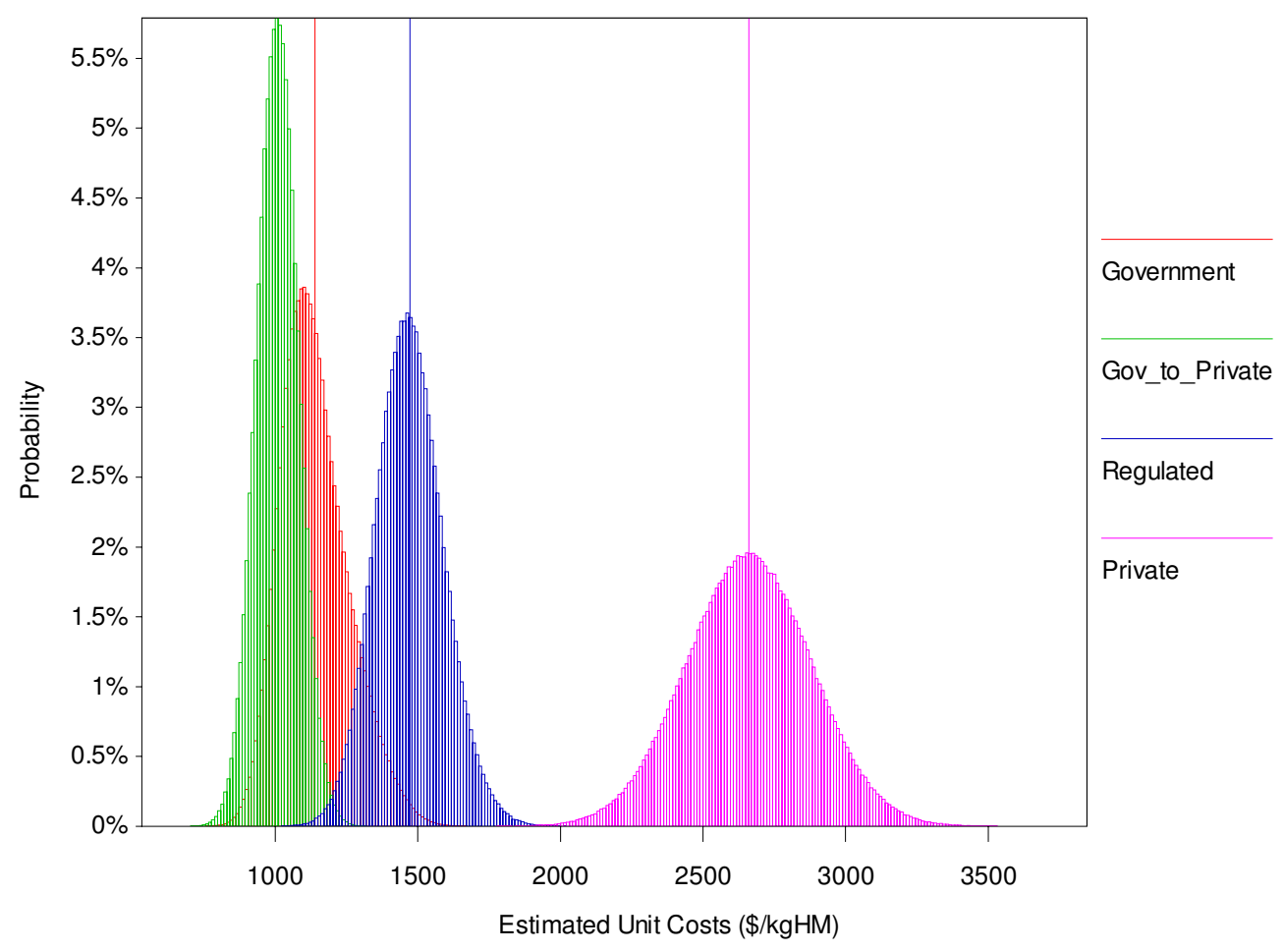

Figure 7. Estimated unit costs in the government-not-forgiving-debt simulation.

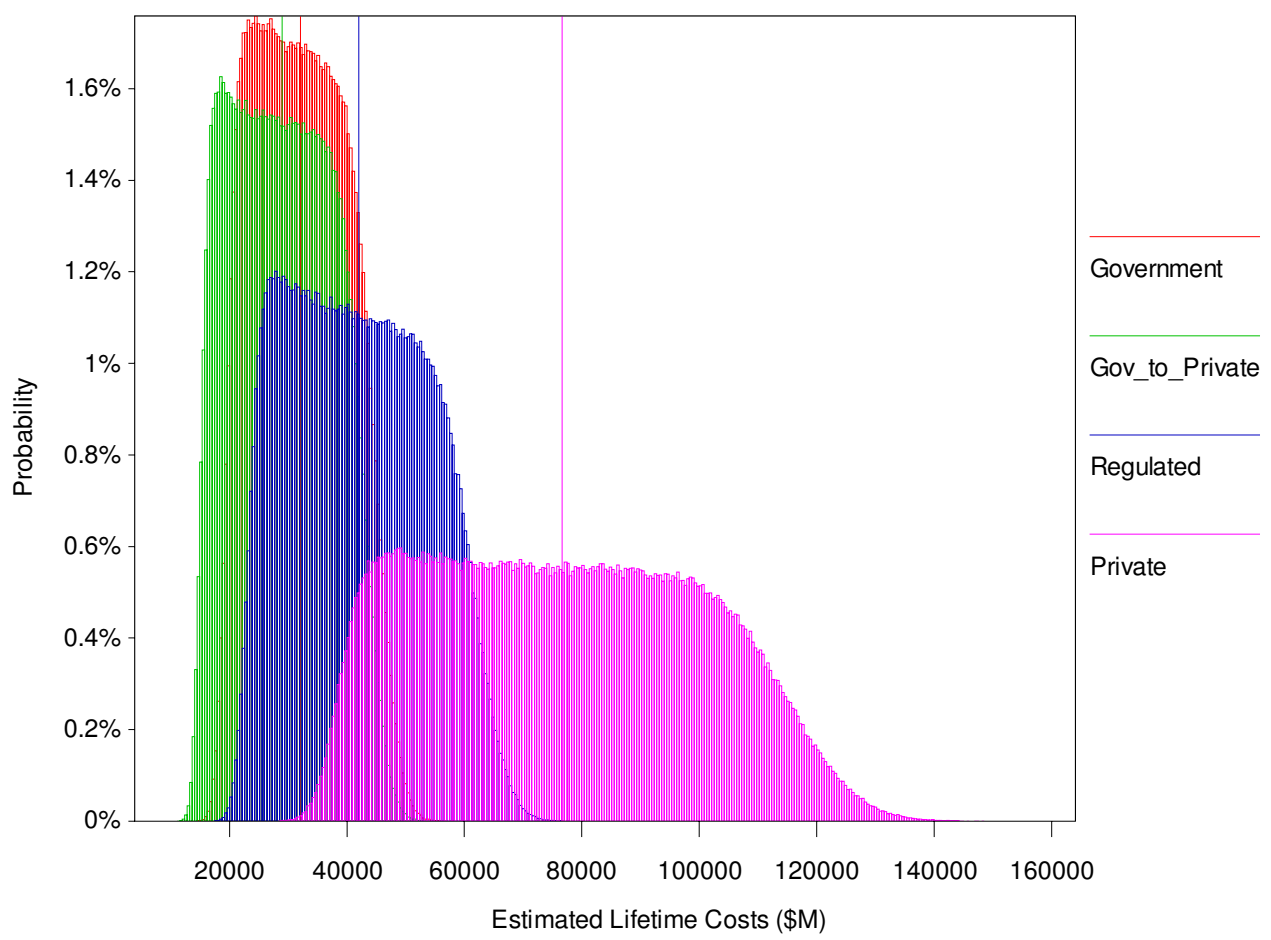

Figure 8. Estimated lifetime costs in government-not-forgiving-debt simulation. 


\subsection{What if operational problems cause the capacity to become less than nominal?}

For this question, the simulation runs with the annual capacity at $30-70 \%(240,000-560,000$ $\mathrm{kgHM} / \mathrm{year}$ ) of the nominal design capacity as a uniform distribution of probability.

The recycling facility may run at lower than the nominal design capacity as a first-of-a-kind facility. Lowering the annual capacity leads to higher unit costs, as should be expected (Figure 9). What might not have been expected is the shape of the new probability distribution graphs. The ranges are much broader, compared to the nominal values, and there is more uncertainty associated with the higher costs in the ranges. An interesting result of this analysis is the asymmetric distribution of the cost curve when the capacity is reduced. The left side of the distribution corresponds to the $70 \%$ value $(560,000$ $\mathrm{kgHM} / \mathrm{year})$ of the capacity reduction and the $30 \%(240,000 \mathrm{kgHM} / \mathrm{year})$ value results in the long distribution tail to the right. The asymmetric cost distribution pattern is derived from the ratio of the fixed (capital recovery) costs to the variable (production) costs for this case.

From Section 2, we learned that the capacity variable has high cost sensitivity. From the tornado diagram in Figure 2, we see that by halving the facility capacity to $400,000 \mathrm{kgHM} / \mathrm{year}$ the unit costs double (an increase in unit costs of $\sim \$ 1193 / \mathrm{kgHM}$ ). Conversely if the capacity is doubled to $1,600,000$ $\mathrm{kgHM} /$ year, then the unit costs are reduced by $50 \%$ (a decrease in unit costs of $\sim \$ 636 / \mathrm{kgHM}$ ). In other words, the costs are more sensitive to capacity decreases than capacity increases.

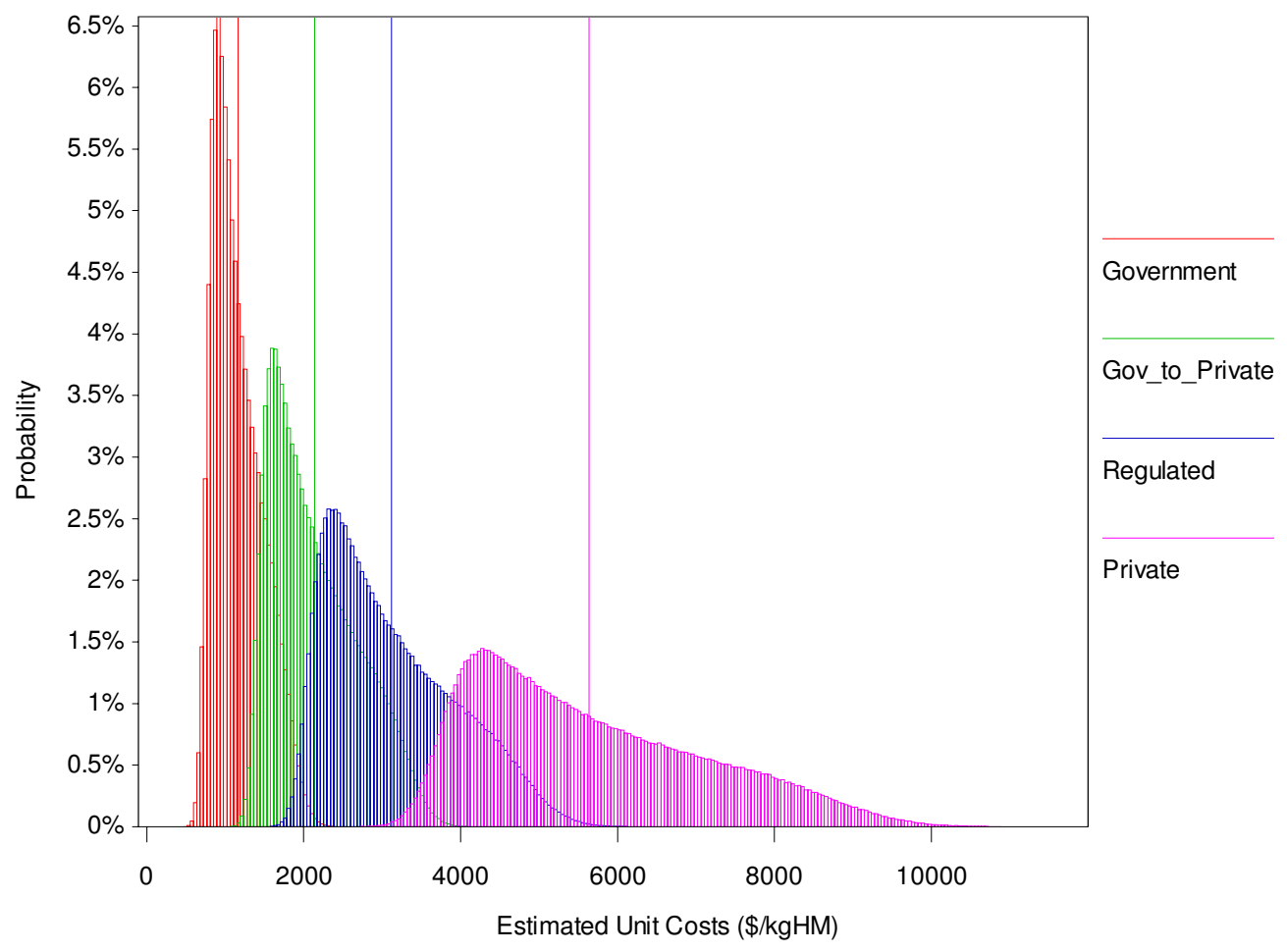

Figure 9. Estimated unit costs in 30-70\% annual capacity simulation. 


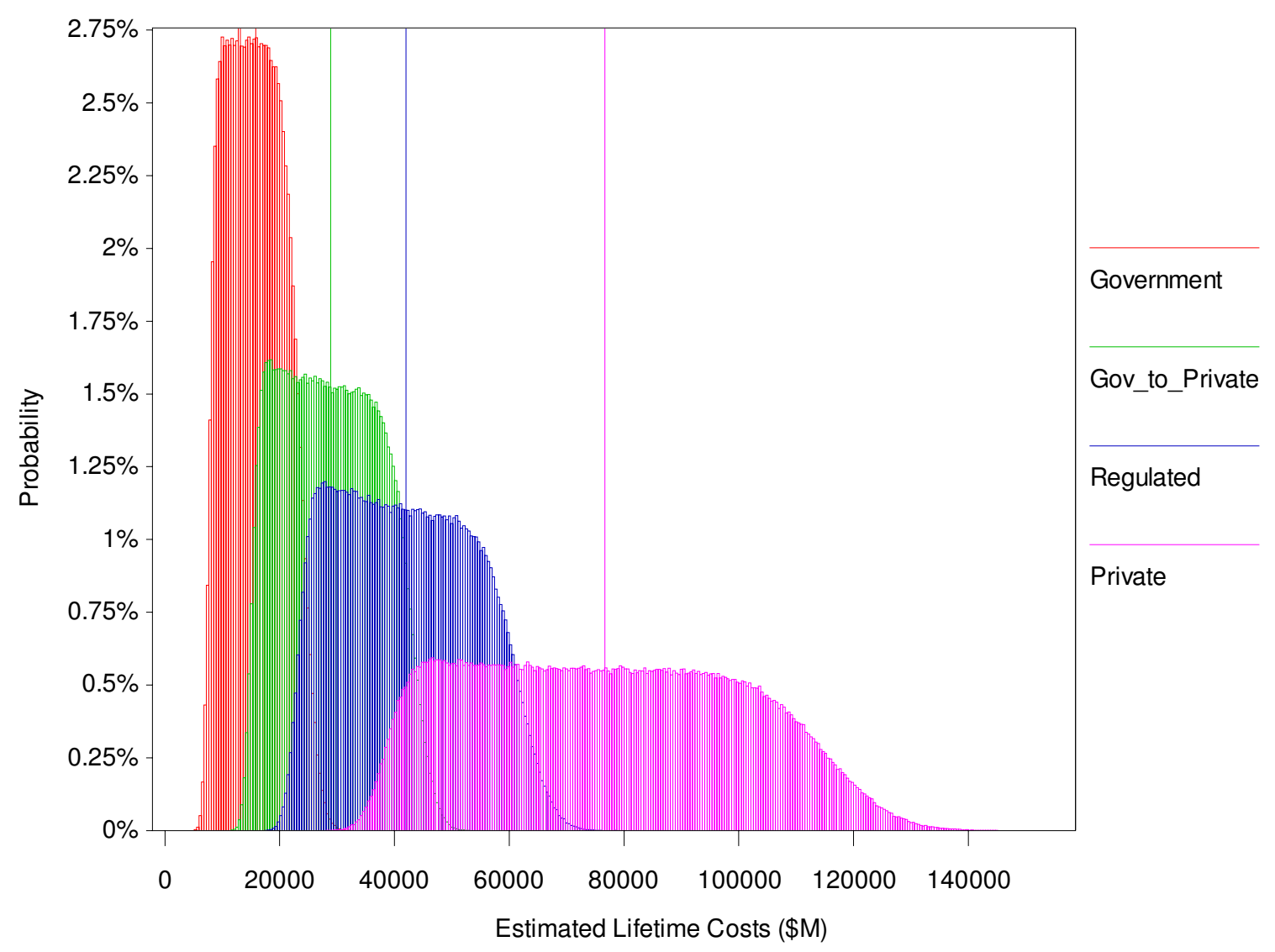

Figure 10. Estimated lifetime costs in 30-70\% annual capacity simulation.

Figure 10 shows an unremarkable result, as the lifetime costs are the same as in the nominal case. This is not necessarily a realistic result, but it follows since the model capital costs and operating costs are derived independent of actual capacity. Were a facility to operate at lower than nominal capacity in the real world, it is not expected that they would have the foresight to exactly recover their costs as modeled here. While the lifetime costs remain unchanged in this result, the overall efficiency of the facility is far lower.

\subsection{What if guaranteed contracts or government incentives lead to lower risk for private investors?}

To run a simulation of lower risk, the debt interest rate and required return on investment were lowered for private investing to 6 and 10\%, respectively. We assume that lowering the risk will bring private ownership closer to the results from regulated ownership. Simulating lower risk is consistent with many proposed legislation ideas to reduce risks for capital intensive energy industry components. At the moment, recycle facilities are not considered in the legislation, but it is possible that the incentives put into place for energy producing facilities might spill over into other types of facilities that support the U.S. energy infrastructure. 
December 16, 2005

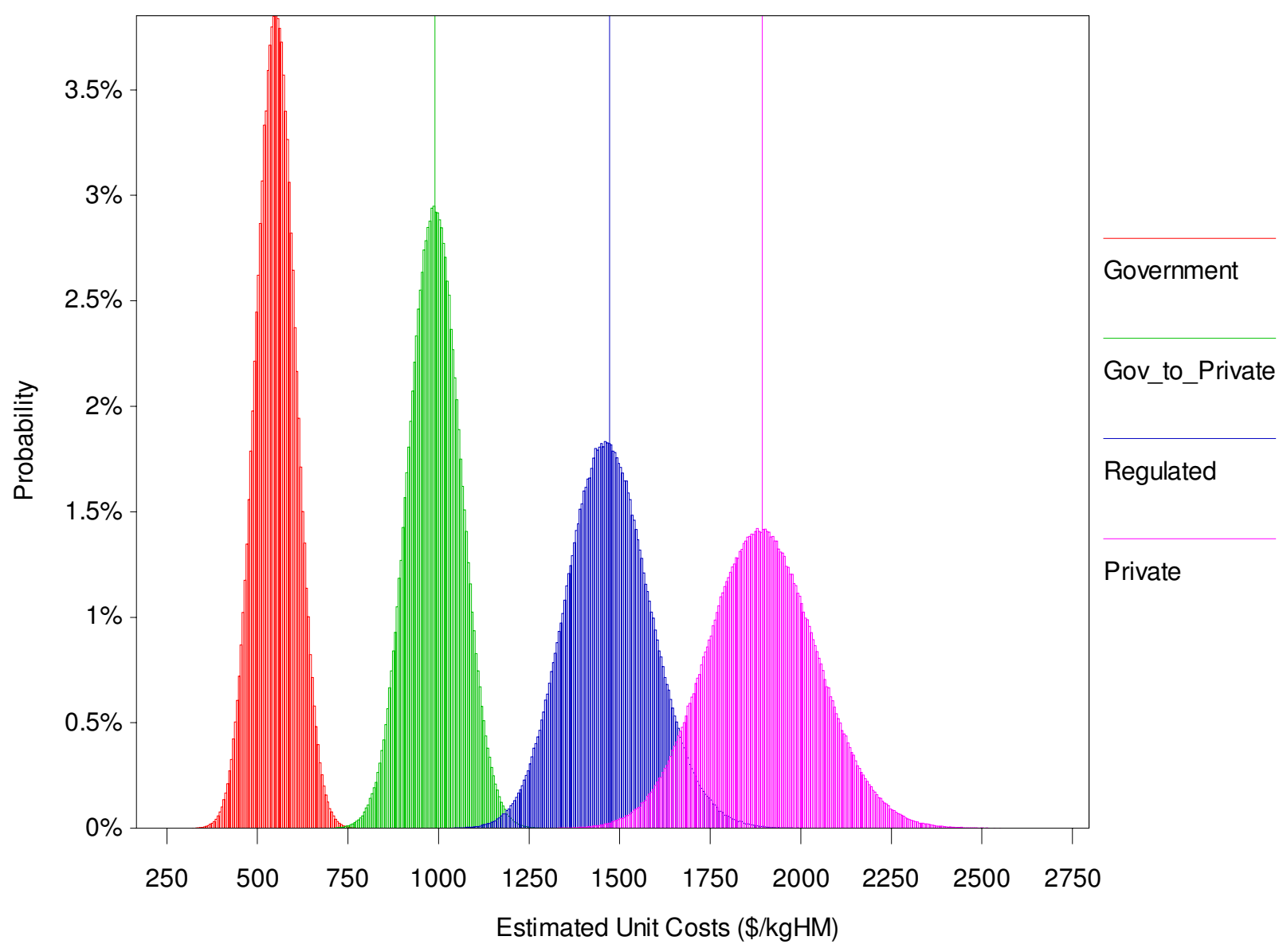

Figure 11. Estimated unit costs in lower risk simulation.

The reduced-risk to the private sector scenario, shown in Figure 11, leads to the private option having improved certainty and lower unit costs. The government-to-private option also has slight improvements in certainty and costs due to the reduction of $0.5 \%$ of the ROI for the equity financing received. 


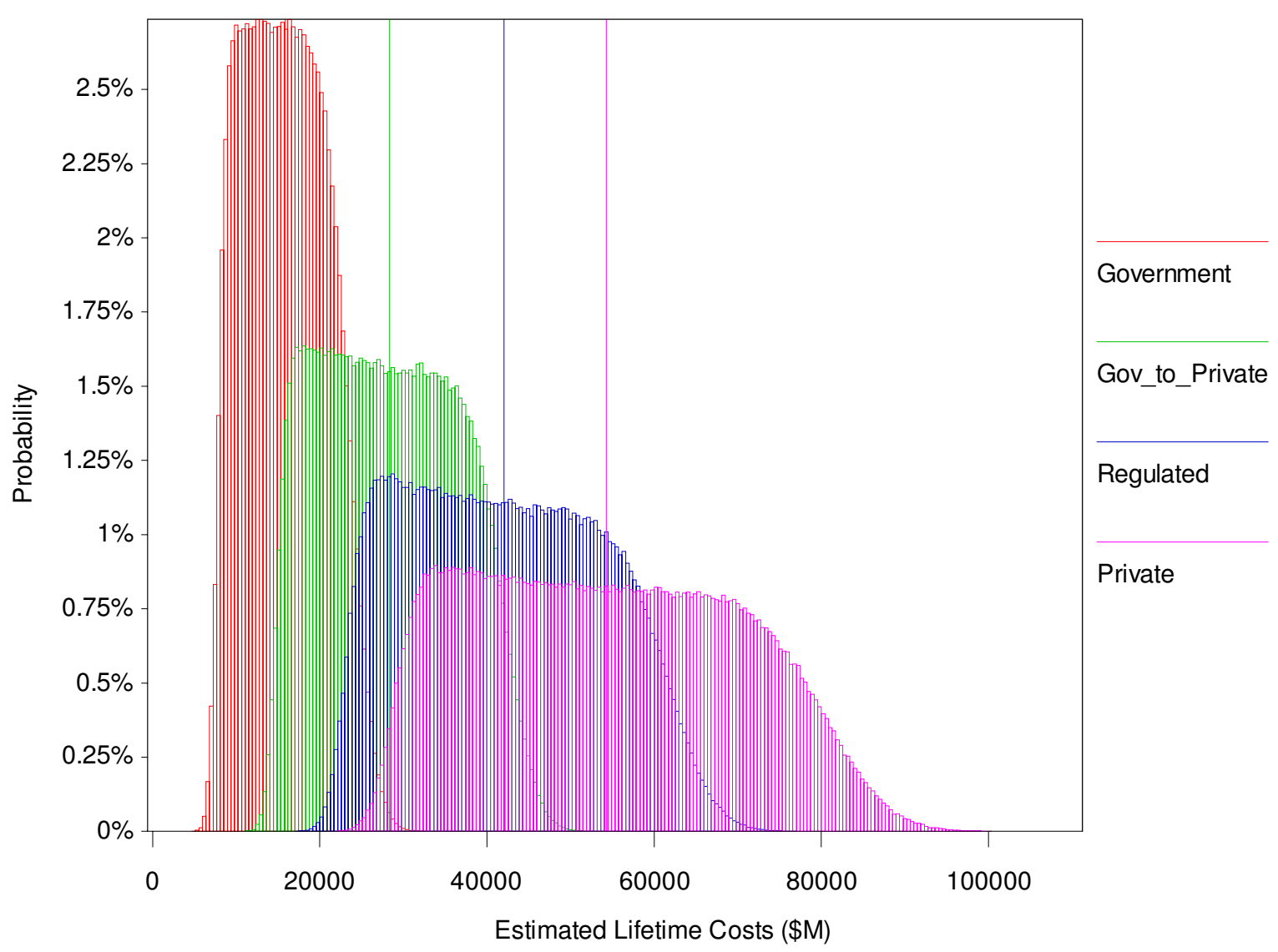

Figure 12. Estimated lifetime costs in the lower risk simulation.

The lifetime cost result in Figure 12 is similar to the unit cost result in Figure 11, with the nonregulated option moving noticeably and the government-to-private moving slightly to the left (decreasing costs). The differences in their movements are due to the original assumptions which already benefited the government-to-private option with a debt interest rate of $4 \%$ and equity ROI of $10.5 \%$. Therefore the government-to-private scenario only gains $0.5 \%$ on the equity portion of its $32.5 \%$ of debt, which results in a very small change in the resulting cost. However the private option benefits by the reduction of the debt interest rate from $9 \%$ to $6 \%$ and equity ROI from $16 \%$ to $10 \%$.

\subsection{How does this analysis incorporating elements of variable uncertainty compare to the original AFCl deterministic analysis?}

In comparison between the original recycling facility financing study prepared in FY-05 and this analysis, we have begun to identify a range of options that could result in lower recycle facility costs. Most importantly, we have shown the importance of operating capacity and uncertainty in determining unit and lifetime costs. We have also shown the limitations of the deterministic approach for selecting financing alternatives, and the value of understanding cost uncertainty. We have brought focus to the advantages from economies of scale from producing higher capacity facilities, where the largest portion of the cost is in the facility construction. 


\section{CONCLUSIONS}

The most consistent result from these studies is that government ownership leads to lowest costs. However, this does not mean that the facilities should necessarily be constructed and operated by the government. Through this analysis we found that the costs of the government-to-private and the private (regulated) options were not greatly different than the fully government option. This analysis indicates that commercial operations have potential to be economical, but there is presently no incentive for private industry involvement, since the current legal framework establishes government ownership of partially used fuel (OCRWM/DOE 2004).

We have come to similar conclusions regarding the significance of cost variables as does the AFCI 2005 study prepared by Chaim Braun. In the government ownership option; annual O\&M costs, forgiveness of debt and overnight costs are the most significant. In the regulated and private cases, initial capital costs will be most significant. Further, we have established that uncertainty in variables has a greater effect on the private ownership option than the others.

Uncertainty in operation, leading to lower annual capacity, is the most detrimental scenario economically. Determining the facilities throughput will be a driving factor in setting unit costs. There is a significant penalty for operating at less than capacity, as shown by the 30 to $70 \%$ nominal annual capacity simulation. With appreciable economies for full-capacity facility operation, the facility should be designed for maximum throughput and availability to support the needs of the nuclear power plant fleet. If high capacities (approaching 3,000 MT/year) are obtained within the same capital and operation cost parameters as modeled, then unit costs as low as $\$ 500 / \mathrm{kgHM}$ could be achieved. These capacities could result from significant economies of scale (specifically capital cost versus capacity) arising from the construction design and facility operations designed for optimal economic throughput.

Mitigating risks, thereby lowering debt interest rates and required return on investments, led to the most promising result for private industry initiative. Most of the costs for the private ventures are found in the interest on debt and equity for the initial capital investment. Any mechanism causing the capital investment and the associated returns to decrease will lead to a more attractive situation for private ownership.

Additional studies on facility ownership will be researched and incorporated into this draft for the 2006 AFC Cost Basis report. The authors are aware of similar studies on MOX fuel fabrication facilities (ORNL 1996) for weapons plutonium disposition. These studies showed that government ownership lead to the lowest life cycle and unit costs. For the U.S. MOX Fabrication Facility, now under construction at the Savannah River site, government funds are being used for construction; however, a private company will operate the plant with performance-based incentives. Also additional analysis will be performed to better understand facility capacity cost sensitivities and other high cost impact items. 


\section{REFERENCES}

AFCI 2005, Advanced Fuel Cycle Initiative: Objectives, Approach, and Technology Summary. U.S. Department of Energy Office of Nuclear Energy, Science, and Technology. May 2005. http://www.ne.doe.gov/reports/AFCI_RptCong_ObjApp\%5BTechSummMay2005.pdf.

Bunn M., S. Fetter, J. P. Holdren, and R. van der Zwan,, 2003, The Economics of Reprocessing vs. Direct Disposal of Spent Nuclear Fuel, Section A.2.1.2. Nuclear Technology 150:3 209-230.

Droge and Schroder, 2005, How to turn and industry green: Taxes versus Subsidies. Journal of Regulatory Economics 27:2 177-202.

Gingold, J. E., R. W. Kupp, D. Schaeffer, and R. L. Klein, 1991. The Cost of Processing Irradiated Fuel From Light Water Reactors: An Independent Assessment. EPRI NP-7264. Palo Alto, Calif.: EPRI.

Kwoka, John Jr., 2005, The Comparative Advantage of Public Ownership: Evidence from U.S. Electric Utilities. Canadian Journal of Economics 38:2 622-640.

National Research Council, 1996, Nuclear Wastes: Technologies for Separation and Transmutation, Appendix J. Commission on Geosciences, Environment and Resources. Washington D.C.: National Academies Press.

Office of Civilian Radioactive Waste Management/Department of Energy (OCRWM/DOE), 2004. Nuclear Waste Policy Act as amended. Washington D.C.: OCRWM/DOE. http://www.ocrwm.doe.gov/documents/nwpa/css/nwpa.htm.

Organization for Economic Cooperation And Development/Nuclear Energy Agency (OECD/NEA), 1993. The Economics of the Nuclear Fuel Cycle. OECD/NEA, Paris.

Organization for Economic Cooperation And Development/Nuclear Energy Agency (OECD/NEA), 2002, Trends in the Nuclear Fuel Cycle: Economic, Environmental, and Social Aspects. OECD/NEA, Paris.

D. Shropshire, et al, 2005, 2005 Advanced Fuel Cycle Cost Basis, INEEL/EXT-04-02282, July 2005, DRAFT Rev. A. Official Use Only under FOIA exemption 5. 